\title{
Research Article \\ Exponential Stability of Impulsive Stochastic Functional Differential Systems
}

\author{
Zheng Wu, Hao Huang, and Lianglong Wang \\ School of Mathematical Science, Anhui University, Anhui, Hefei 230039, China \\ Correspondence should be addressed to Lianglong Wang, wangll@ahu.edu.cn \\ Received 2 January 2012; Revised 25 February 2012; Accepted 26 February 2012 \\ Academic Editor: Josef Diblík
}

Copyright (C) 2012 Zheng Wu et al. This is an open access article distributed under the Creative Commons Attribution License, which permits unrestricted use, distribution, and reproduction in any medium, provided the original work is properly cited.

This paper is concerned with stabilization of impulsive stochastic delay differential systems. Based on the Razumikhin techniques and Lyapunov functions, several criteria on $p$ th moment and almost sure exponential stability are established. Our results show that stochastic functional differential systems may be exponentially stabilized by impulses.

\section{Introduction}

In the past decades, many authors have obtained various results of deterministic functional differential systems (see [1-6] and the references therein). But it is well known that there are many stochastic factors in the realistic environment, and it is necessary to consider stochastic models. In fact, stochastic functional differential systems (SFDSs) have received more attention in recent years. The properties of SFDSs including stability have been studied in [7-10], which can be widely used in science and engineering (see [11] and the references therein). Furthermore, besides stochastic effects, impulsive effects likewise exist in many evolution processes in which system states change abruptly at certain moments of time, involving such fields as medicine and biology, economics, mechanics, electronics, and telecommunications, and so forth. The impulsive control theory comes to play an important role in science and industry [12]. So the stability investigation of impulsive stochastic differential systems (ISDSs) and impulsive stochastic functional differential systems (ISFDSs) is interesting to many authors [13-20].

Recently, the Razumikhin-type asymptotical stability theorems for ISFESs were established [21, 22]. However, little work has been done on generally exponential stability of ISFESs $[23,24]$. In this paper, stability criteria for impulsive stochastic function differential systems are investigated by Razumikhin technique and Lyapunov functions. It is shown that 
an unstable stochastic delay system can be successfully stabilized by impulses and the results can be easily applied.

\section{Preliminaries}

Throughout this paper, unless otherwise specified, let $\left(\Omega, \mathcal{F},\left\{\mathcal{F}_{t}\right\}_{t \geq 0}, P\right)$ be a complete probability space with a filtration $\left\{\mathscr{F}_{t}\right\}_{t \geq 0}$ satisfying the usual conditions (i.e., it is right continuous and $\mathcal{F}_{0}$ contains all $P$-null sets). $w(t)=\left(w_{1}(t), w_{2}(t), \ldots, w_{d}(t)\right)^{T}$ means a $d$ dimensional Brownian motion defined on this probability space. $R$ denotes the set of real numbers, $R_{+}$is the set of nonnegative real numbers, and $R^{n}$ denotes the $n$-dimensional real space equipped with Euclidean norm $|\cdot|$. If $A$ is a vector or matrix, its transpose is denoted by $A^{T}$ and its operator norm is denoted by $\|A\|=\sup \{|A x|:|x|=1\}$. Moreover, let $\tau>0$ and denote by $C\left([-\tau, 0] ; R_{+}\right)$the family of continuous functions from $[-\tau, 0]$ to $R_{+}$. Let $N$ denote the set of positive integers, that is, $N=\{1,2, \ldots\}$.

For $-\infty<a<b<+\infty$, a function from $[a, b]$ to $R^{n}$ is called piecewise continuous, if the function has at most a finite number of jump discontinuities on $(a, b]$, which is continuous from the right for all points in $[a, b)$. Given $\tau>0, P C\left([-\tau, 0] ; R^{n}\right)$ denotes the family of piecewise continuous functions from $[-\tau, 0]$ to $R^{n}$. A norm on $P C\left([-\tau, 0] ; R^{n}\right)$ is defined as $\|\phi\|=\sup _{-\tau \leq s \leq 0}|\phi(s)|$ for $\phi \in P C\left([-\tau, 0] ; R^{n}\right)$.

For $p>0$ and $t \geq 0$, let $P C_{\mathcal{F}_{t}}^{p}\left([-\tau, 0] ; R^{n}\right)$ denote the family of all $\mathcal{F}_{t^{-}}$ measurable $P C\left([-\tau, 0] ; R^{n}\right)$-value random variables $\phi$ such that $\sup _{-\tau \leq \theta \leq 0} E|\phi(\theta)|^{p}<\infty$ and $P C_{q_{t}}^{b}\left([-\tau, 0] ; R^{n}\right)$ denote the family of $P C\left([-\tau, 0] ; R^{n}\right)$-value random variables that are bounded and $\mathcal{F}_{t}$-measurable.

In this paper, we consider the following ISFDS:

$$
\begin{gathered}
d x(t)=f\left(x_{t}, t\right) d t+g\left(x_{t}, t\right) d w(t), \quad t \neq t_{k}, t \geq t_{0}, \\
\Delta x\left(t_{k}\right)=I_{k}\left(x_{t_{k^{\prime}}}, t_{k}\right), \quad k \in N \\
x_{t_{0}}=\xi
\end{gathered}
$$

where the initial value $\xi \in P C_{\Psi_{t_{0}}}^{b}\left([-\tau, 0] ; R^{n}\right), x(t)=\left(x_{1}(t), \ldots, x_{n}(t)\right)^{T}, x_{t}$ is regarded as a $P C\left([-\tau, 0] ; R^{n}\right)$-value process and $x_{t}(\theta)=x(t+\theta), \theta \in[-\tau, 0]$. Similarly, $x_{t^{-}}$is defined by $x_{t^{-}}(\theta)=x(t+\theta), \theta \in[-\tau, 0)$ and $x_{t^{-}}(0)=\lim _{s \rightarrow t^{-}} x(s)$. Both $f: P C_{\mathcal{F}_{t}}^{b}\left([-\tau, 0] ; R^{n}\right) \times R_{+} \rightarrow$ $R^{n}$ and $g: P C_{\Psi_{t}}^{b}\left([-\tau, 0] ; R^{n}\right) \times R_{+} \rightarrow R^{n \times d}$ are Borel measurable, and $I_{k}: P C_{\Psi_{t}}^{b}\left([-\tau, 0] ; R^{n}\right) \times$ $R_{+} \rightarrow R^{n}$ represents the impulsive perturbation of $x$ at time $t_{k}$. The fixed moments of impulse times $t_{k}$ satisfy $0 \leq t_{0}<t_{1}<\cdots<t_{k}<\cdots, t_{k} \rightarrow \infty($ as $k \rightarrow \infty), \Delta x\left(t_{k}\right)=x\left(t_{k}\right)-x\left(t_{k}^{-}\right)$. Moreover, $f, g$, and $I_{k}$ are assumed to satisfy necessary assumptions so that, for any initial data $\xi \in P C_{\Phi_{t_{0}}}^{b}\left([-\tau, 0] ; R^{n}\right)$, system $(2.1)$ has a unique global solution, denoted by $x\left(t ; t_{0}, \xi\right)$ (e.g., see [25] for existence and uniqueness results for general impulsive hybrid stochastic delay systems including (2.1)). For the purpose of stability in this note, we also assume the $f(0, t) \equiv 0, g(t, 0) \equiv 0$ and $I_{k}(0, t) \equiv 0$ for all $t \geq t_{0}, k \in N$, then system (2.1) admits a trivial solution.

Definition 2.1. The trivial solution of system (2.1) is said to be $p$ th $(p>0)$ moment exponentially stable if there is a pair of positive constants $\lambda, C$ such that

$$
E\left|x\left(t ; t_{0}, \xi\right)\right|^{p} \leq C\|\xi\|^{p} e^{-\lambda\left(t-t_{0}\right)}, \quad t \geq t_{0}
$$


for all $\xi \in P C_{\Phi_{t_{0}}}^{b}\left([-\tau, 0] ; R^{n}\right)$. When $p=2$, it is usually said to be exponentially stable in mean square. It follows from (2.2) that

$$
\limsup _{t \rightarrow \infty} \frac{1}{t} \log E\left|x\left(t ; t_{0}, \xi\right)\right|^{p} \leq-\lambda
$$

The left-hand side of (2.3) is called the $p$ th moment Lyapunov exponent of the solution.

Definition 2.2. The trivial solution of system (2.1) is said to be almost exponentially stable if there is a pair of positive constants $\lambda, C$ such that for $t \geq t_{0}$

$$
\left|x\left(t ; t_{0}, \xi\right)\right|^{p} \leq C\|\xi\| e^{-\lambda\left(t-t_{0}\right)}, \quad \text { a.s., }
$$

for all $\xi \in P C_{\Psi_{t_{0}}}^{b}\left([-\tau, 0] ; R^{n}\right)$. It follows from (2.4) that

$$
\limsup _{t \rightarrow \infty} \frac{1}{t} \log \left|x\left(t ; t_{0}, \xi\right)\right| \leq-\lambda
$$

The left-hand side of (2.5) is called the Lyapunov exponent of the solution.

Definition 2.3. Let $C^{2,1}\left(R^{n} \times\left[t_{0}, \infty\right) ; R_{+}\right)$denote the family of all nonnegative functions $V(x, t)$ on $R^{n} \times\left[t_{0}-\tau, \infty\right)$ that are continuously twice differential in $x$ and once in $t$. If $V \in C^{2,1}\left(R^{n} \times\right.$ $\left.\left[t_{0}, \infty\right) ; R_{+}\right)$, define the operator $\mathcal{L} V: P C\left([-\tau, 0] ; R^{n}\right) \times\left[t_{0}, \infty\right) \rightarrow R$ for system $(2.1)$ by

$$
\mathcal{L V}\left(x_{t}, t\right)=V_{t}(x, t)+V_{x}(x, t) f\left(x_{t}, t\right)+\frac{1}{2} \operatorname{trace}\left[g^{T}\left(x_{t}, t\right) V_{x x}(x, t) g\left(x_{t}, t\right)\right]
$$

where $V_{t}(x, t)=\partial V(x, t) / \partial t, V_{x}(x, t)=\left(\partial V(x, t) / \partial x_{1}, \ldots, \partial V(x, t) / \partial x_{n}\right), V_{x x}(x, t)=$ $\left(\partial^{2} V(x, t) / \partial x_{i} \partial x_{j}\right)_{n \times n}$.

\section{Main Results}

In this section, we will establish some criteria on the $p$ th moment exponential stability and almost exponential stability for system (2.1) by using the Razumikhin technique and Lyapunov functions. We begin with the following lemma, which concerns with the continuity of $E V(x(t), t)$.

Lemma 3.1. Let $V(x, t) \in C^{2,1}\left(R^{n} \times\left[t_{0}, \infty\right) ; R_{+}\right)$, and let $x(t)$ be a solution of system (2.1). If there exists $c>0$ such that $V(x, t) \leq c|x|^{p}$, then $E V(x(t), t)$ is continuous on $\left[t_{k-1}, t_{k}\right), k \in N$.

Proof. By the Itô formula,




for all $t \in\left[t_{k-1}, t_{k}\right)$, where $k \in N$. Since $x_{t_{k-1}} \in P C_{\Psi_{t_{k-1}}}^{b}\left([-\tau, 0] ; R^{n}\right)$, we can find an integer $l_{0}$ such that $\left\|x_{t_{k-1}}\right\|<l_{0}$ a.s. For any integer $l>l_{0}$, define the stopping time

$$
\rho_{l}=\inf \left\{t \in\left[t_{k-1}, t_{k}\right):|x(t)| \geq l\right\},
$$

where $\inf \emptyset=\infty$ as usual. Since $x(t)$ is continuous on $\left[t_{k-1}, t_{k}\right),|x(t)|$ is also continuous on $\left[t_{k-1}, t_{k}\right)$. Clearly, $\rho_{l} \rightarrow \infty$ a.s. as $l \rightarrow \infty$. Moreover, it has $E V\left(x\left(t_{k-1}\right), t_{k}\right) \leq c l_{0}$, following from $x_{t_{k-1}} \in P C_{q_{t_{k-1}}}^{b}\left([-\tau, 0] ; R^{n}\right)$. It then follows from the definition of $\rho_{l}$ above that

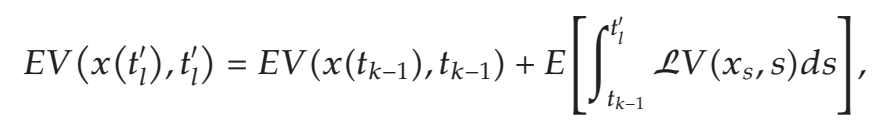

where $t_{l}^{\prime}=t \wedge \rho_{l}$. So, letting $l \rightarrow \infty$, by the dominated convergence theorem and Fubini's theorem, we have

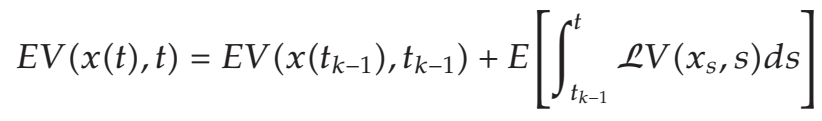

$$
\begin{aligned}
& =E V\left(x\left(t_{k-1}\right), t_{k-1}\right)+\int_{t_{k-1}}^{t} E\left[\mathcal{L} V\left(x_{s}, s\right)\right] d s,
\end{aligned}
$$

for $t \in\left[t_{k-1}, t_{k}\right)$. This implies that $E V(x(t), t)$ is continuous on $\left[t_{k-1}, t_{k}\right), k \in N$.

Theorem 3.2. Let $V \in C^{2,1}\left(R^{n} \times\left[t_{0}-\tau, \infty\right) ; R_{+}\right)$and $u:\left[t_{0}, \infty\right) \rightarrow R_{+}$be a piecewise continuous function. Suppose there exist some positive constants $p, c_{1}, c_{2}$, and $\lambda$ such that

(i) for all $(x, t) \in R^{n} \times\left[t_{0}-\tau, \infty\right)$,

$$
c_{1}|x|^{p} \leq V(x, t) \leq c_{2}|x|^{p},
$$

(ii) for all $k \in N$, and $\phi \in P C_{q_{t}}^{p}\left([-\tau, 0] ; R^{n}\right)$,

$$
E V\left(\phi\left(0^{-}\right)+I\left(t_{k}, \phi\right), t_{k}\right) \leq d_{k} E V\left(\phi\left(0^{-}\right), t_{k}^{-}\right),
$$

where $0<d_{k}<\exp \left\{-\lambda\left(t_{k+1}-t_{k}\right)-\int_{t_{k}}^{t_{k+1}} u(s) d s\right\}$,

(iii) for all $t \geq t_{0}, t \neq t_{k}, k \in N$ and $\phi \in P C_{q_{t}}^{p}\left([-\tau, 0] ; R^{n}\right)$,

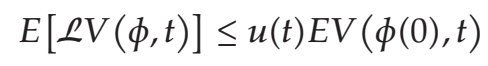

whenever

$$
E V(\phi, t+\theta)<q E V(\phi(0), t), \quad \theta \in[-\tau, 0],
$$

where $q>\max _{k \in N}\left\{d_{k}^{-1} e^{\lambda \tau}\right\} \vee \exp \left\{\int_{t_{0}}^{t_{1}} u(s) d s\right\}$. 
Then the trivial solution of system (2.1) is $p$ th moment exponentially stable and its $p$ th moment Lyapunov exponent is not greater than $-\lambda$.

Proof. Given any initial data $\xi \in P C_{\Phi_{t_{0}}}^{b}\left([-\tau, 0] ; R^{n}\right)$, the global solution $x\left(t ; t_{0}, \xi\right)=x(t)$ of $(2.1)$ is written as $x(t)$ in this proof. Without loss of generality, assume that the initial date $\xi$ is nontrivial so that $x(t)$ is not a trivial solution. Choose $M$ such that

$$
c_{2} e^{\lambda\left(t_{1}-t_{0}\right)+\int_{t_{0}}^{t_{1}} u(s) d s}<M<c_{2} q e^{\lambda\left(t_{1}-t_{0}\right)} .
$$

Then it follows from condition (i) and (3.9) that

$$
E V(x(t), t) \leq c_{2}\|\xi\|^{p}<M\|\xi\|^{p} e^{-\lambda\left(t_{1}-t_{0}\right)}, \quad t \in\left[t_{0}-\tau, t_{0}\right] .
$$

In the following, we will show that

$$
E V(x(t), t) \leq M\|\xi\|^{p} e^{-\lambda\left(t_{k}-t_{0}\right)}, \quad t \in\left[t_{k-1}, t_{k}\right), k \in N .
$$

In order to do so, we first prove that

$$
E V(x(t), t) \leq M\|\xi\|^{p} e^{-\lambda\left(t_{1}-t_{0}\right)}, \quad t \in\left[t_{0}, t_{1}\right) .
$$

If (3.12) is not true, then there exist some $t \in\left[t_{0}, t_{1}\right)$ such that $E V(x(t), t)>M\|\xi\|^{p} e^{-\lambda\left(t_{1}-t_{0}\right)}$. Set $t^{*}=\inf \left\{t \in\left[t_{0}, t_{1}\right): E V(x(t), t)>M\|\xi\|^{p} e^{-\lambda\left(t_{1}-t_{0}\right)}\right\}$. Then $t^{*} \in\left(t_{0}, t_{1}\right)$ and also, by the continuity of $E V(x(t), t)$ ) (see Lemma 3.1),

$$
E V(x(t), t)<E V\left(x\left(t^{*}\right), t^{*}\right)=M\|\xi\|^{p} e^{-\lambda\left(t_{1}-t_{0}\right)}, \quad t \in\left[t_{0}-\tau, t_{*}\right) .
$$

In view of (3.10), define $t_{*}=\sup \left\{t \in\left[t_{0}-\tau, t^{*}\right): E V(x(t), t) \leq c_{2}\|\xi\|^{p}\right\}$. Then $t_{*} \in\left[t_{0}, t^{*}\right)$ and, by the continuity of $E V(x(t), t)$,

$$
E V(x(t), t)>E V\left(x\left(t_{*}\right), t_{*}\right)=c_{2}\|\xi\|^{p}, \quad t \in\left(t_{*}, t^{*}\right] .
$$

Now in view of (3.9), (3.13), and (3.14), one has, for $t \in\left[t_{*}, t^{*}\right]$ and $\theta \in[-\tau, 0]$,

$$
E V(x(t+\theta), t+\theta) \leq M\|\xi\|^{p} e^{-\lambda\left(t_{1}-t_{0}\right)}<q E V\left(x\left(t_{*}\right), t_{*}\right) \leq q E V(x(t), t) .
$$

By the Razumikhin-type condition (iii),

$$
E\left[\mathcal{L} V\left(x_{t}, t\right)\right] \leq u(t) E V(x(t), t), \quad \forall t \in\left[t_{*}, t^{*}\right] .
$$

Applying Itô formula and by (3.16), one obtains that

$$
E V\left(x\left(t^{*}\right), t^{*}\right) \leq E V\left(x\left(t_{*}\right), t_{*}\right)+\int_{t_{*}}^{t^{*}} u(s) E V(x(s), s) d s .
$$


Finally, by (3.9), (3.13), (3.14), and the Gronwall inequality,

$$
\begin{aligned}
E V\left(x\left(t^{*}\right), t^{*}\right) & \leq E V\left(x\left(t_{*}\right), t_{*}\right) e^{\int_{t_{*}}^{t_{*}^{*}} u(s) d s} \leq c_{2}\|\xi\|^{p} e^{\int_{t_{0}}^{t_{1}} u(s) d s} \\
& <M\|\xi\|^{p} e^{-\lambda\left(t_{1}-t_{0}\right)}=E V\left(x\left(t^{*}\right), t^{*}\right)
\end{aligned}
$$

which is a contradiction. So inequality (3.12) holds and (3.11) is true for $k=1$.

Now assume that

$$
E V(x(t), t) \leq M\|\xi\|^{p} e^{-\lambda\left(t_{k}-t_{0}\right)}, \quad \forall t \in\left[t_{k-1}, t_{k}\right), k \in N,
$$

for all $k \leq m$, where $k, m \in N$. We proceed to show that

$$
E V(x(t), t) \leq M\|\xi\|^{p} e^{-\lambda\left(t_{m+1}-t_{0}\right)}, \quad \forall t \in\left[t_{m}, t_{m+1}\right)
$$

Suppose (3.20) is not true, set $\bar{t}=\inf \left\{t \in\left[t_{m}, t_{m+1}\right): E V(x(t), t)>M\|\xi\|^{p} e^{-\lambda\left(t_{m+1}-t_{0}\right)}\right\}$. By condition (ii) and (3.20), we know

$$
E V\left(x\left(t_{m}\right), t_{m}\right) \leq d_{m} E V\left(x\left(t_{m}^{-}\right), t_{m}^{-}\right) \leq d_{m} M\|\xi\|^{p} e^{-\lambda\left(t_{m}-t_{0}\right)}<M\|\xi\|^{p} e^{-\lambda\left(t_{m+1}-t_{0}\right)} .
$$

From this, together with $E V(x(t), t)$ being continuous on $t \in\left[t_{m}, t_{m+1}\right)$, we know that $\bar{t} \in$ $\left(t_{m}, t_{m+1}\right)$ and

$$
E V(x(t), t)<E V(x(\bar{t}), \bar{t})=M\|\xi\|^{p} e^{-\lambda\left(t_{m+1}-t_{0}\right)}, \quad \forall t \in\left[t_{m}, \bar{t}\right) .
$$

Define $\underline{t}=\sup \left\{t \in\left[t_{0}, \bar{t}\right]: E V(x(t), t) \leq d_{m} M\|\xi\|^{p} e^{-\lambda\left(t_{m}-t_{0}\right)}\right\}$, then $\underline{t} \in\left[t_{m}, \bar{t}\right)$ and

$$
E V(x(t), t)>E V(x(\underline{t}), \underline{t})=d_{m} M\|\xi\|^{p} e^{-\lambda\left(t_{m}-t_{0}\right)}, \quad \forall t \in(\underline{t}, \bar{t}] .
$$

For $t \in[\underline{t}, \bar{t}]$ and $\theta \in[-\tau, 0]$, when $t+\theta \geq t_{m}$, then (3.22) and (3.23) imply that

$$
\begin{aligned}
E V(x(t+\theta), t+\theta) & \leq M\|\xi\|^{p} e^{-\lambda\left(t_{m+1}-t_{0}\right)}<M\|\xi\|^{p} e^{-\lambda\left(t+\theta-t_{0}\right)} \\
& \leq M e^{\lambda \tau}\|\xi\|^{p} e^{-\lambda\left(t-t_{0}\right)} \leq M e^{\lambda \tau}\|\xi\|^{p} e^{-\lambda\left(t_{m}-t_{0}\right)} \\
& \leq q E V(x(\underline{t}), \underline{t}) .
\end{aligned}
$$

If $t+\theta<t_{m}$ for some $\theta \in[-\tau, 0)$, we assume that, without loss of generality, $t+\theta \in\left[t_{l}, t_{l+1}\right)$ for some $l \in N, l \leq m-1$, then from (3.19) and (3.23),

$$
\begin{aligned}
E V(x(t+\theta), t+\theta) & \leq M\|\xi\|^{p} e^{-\lambda\left(t_{l+1}-t_{0}\right)}<M\|\xi\|^{p} e^{-\lambda\left(t+\theta-t_{0}\right)} \\
& \leq M e^{\lambda \tau}\|\xi\|^{p} e^{-\lambda\left(t-t_{0}\right)} \leq M e^{\lambda \tau}\|\xi\|^{p} e^{-\lambda\left(t_{m}-t_{0}\right)} \\
& \leq q E V(x(\underline{t}), \underline{t}) .
\end{aligned}
$$


Therefore,

$$
E V(x(t+\theta), t+\theta)<q E V(x(t), t), \quad t \in[\underline{t}, \bar{t}], \theta \in[-\tau, 0]
$$

Then, it follows from condition (iii) that

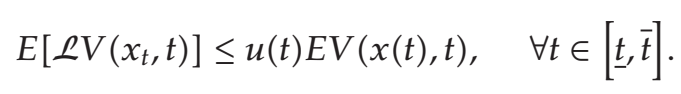

Combining Itô formula with (3.27), we can check that

$$
E V(x(\bar{t}, \bar{t})) \leq E V(x(\underline{t}), \underline{t})+\int_{\bar{t}}^{\underline{t}} u(s) E V(x(s), s) d s
$$

Finally, by (3.22), (3.23), and the Gronwall inequality,

$$
\begin{aligned}
E V(x(\bar{t}), \bar{t}) & \leq E V(x(\underline{t}), \underline{t}) e^{\int_{\underline{t}}^{\bar{t}} u(s) d s} \leq E V(x(\underline{t}), \underline{t}) e^{\int_{t_{m}}^{t_{m+1}} u(s) d s} \\
& =d_{m} M\|\xi\|^{p} e^{-\lambda\left(t_{m}-t_{0}\right)} e^{\int_{t_{m}}^{t_{m+1}} u(s) d s}<E V(x(\bar{t}), \bar{t}),
\end{aligned}
$$

which is a contradiction. So inequality (3.20) holds. By mathematical induction, we obtain that (3.11) holds for all $k \in N$. Furthermore, from condition (i), we have

$$
E|x(t)|^{p} \leq \frac{c_{1}}{c_{2}} M\|\xi\|^{p} e^{-\lambda\left(t_{k}-t_{0}\right)} \leq \frac{c_{1}}{c_{2}} M\|\xi\|^{p} e^{-\lambda\left(t-t_{0}\right)}, \quad t \in\left[t_{k-1}, t_{k}\right), k \in N,
$$

which implies

$$
E\|x\|^{p} \leq \frac{c_{1}}{c_{2}} M\|\xi\|^{p} e^{-\lambda\left(t-t_{0}\right)}, \quad t \geq t_{0}
$$

that is, system (2.1) is $p$ th moment exponentially stable. The proof is complete.

Remark 3.3. If $u(t) \equiv c>0$, then Theorem 3.1 of [23] follows from Theorem 3.2 immediately.

Theorem 3.4. Let $V \in C^{2,1}\left(R^{n} \times\left[t_{0}-\tau, \infty\right) ; R_{+}\right)$, and let $u:\left[t_{0}, \infty\right) \rightarrow R_{+}$be a piecewise continuous function. Suppose there exist some positive constants $p, c_{1}, c_{2}$, and $\lambda$ such that

(i) for all $(x, t) \in R^{n} \times\left[t_{0}-\tau, \infty\right)$,

$$
c_{1}|x|^{p} \leq V(x, t) \leq c_{2}|x|^{p},
$$

(ii) for all $k \in N$ and $\phi \in P C_{\mathcal{F}_{t}}^{p}\left([-\tau, 0] ; R^{n}\right)$,

$$
E V\left(\phi\left(0^{-}\right)+I\left(t_{k}, \phi\right)\right) \leq \rho d_{k} E V\left(\phi\left(0^{-}\right), t_{k}^{-}\right)
$$

where $0<\rho<\max \left\{e^{-\lambda\left(t_{m+1}-t_{m}\right)}\right\}$ and $d_{k}>0$ with $\widehat{d}=\sup _{n \in N} \Pi_{k=1}^{n} d_{k}<\infty$, 
(iii) for all $t \geq t_{0}, t \neq t_{k}, k \in N$, and $\phi \in P C_{q_{t}}^{p}\left([-\tau, 0] ; R^{n}\right)$,

$$
E[\mathcal{L V}(\phi, t)] \leq u(t) E V(\phi(0), t)
$$

whenever

$$
E V(\phi, t+\theta)<q E V(\phi(0), t), \quad \theta \in[-\tau, 0],
$$

where $q>\left(\rho^{-1} e^{\lambda \tau}\right) \vee\left(\rho^{-1} e^{\lambda \tau} \widehat{d}^{-1}\right)$.

Then the trivial solution of system (2.1) is $p$ th moment exponentially stable and its $p$ th moment Lyapunov exponent is not greater than $-\lambda$.

Proof. Given any initial data $\xi \in P C_{\Phi_{t_{0}}}^{b}\left([-\tau, 0] ; R^{n}\right)$, the global solution $x\left(t ; t_{0} ; \xi\right)=x(t)$ of $(2.1)$ is written as $x(t)$ in this proof. Without loss of generality, assume that the initial date $\xi$ is nontrivial so that $x(t)$ is not a trivial solution. Choose $M$ such that

$$
c_{2} e^{\lambda\left(t_{1}-t_{0}\right)+\int_{t_{0}}^{t_{1}} u(s) d s}<M<c_{2} q e^{\lambda\left(t_{1}-t_{0}\right)} .
$$

Then it follows from condition (i) and (3.36) that

$$
E V(x(t), t) \leq c_{2}\|\xi\|^{p}<M\|\xi\|^{p} e^{-\lambda\left(t_{1}-t_{0}\right)}, \quad t \in\left[t_{0}-\tau, t_{0}\right]
$$

In the following, we will show that

$$
E V(x(t), t) \leq M_{k}\|\xi\|^{p} e^{-\lambda\left(t_{k}-t_{0}\right)}, \quad t \in\left[t_{k-1}, t_{k}\right),
$$

where $k \in N$ and $M_{k}$ is defined as $M_{1}=M$ and $M_{k}=M \Pi_{1 \leq l \leq k-1} d_{l}$. Similarly, as the proof in Theorem 3.2, one can prove that

$$
E V(x(t), t) \leq M\|\xi\|^{p} e^{-\lambda\left(t_{1}-t_{0}\right)}, \quad t \in\left[t_{0}, t_{1}\right)
$$

Now assume that

$$
E V(x(t), t) \leq M_{k}\|\xi\|^{p} e^{-\lambda\left(t_{k}-t_{0}\right)}, \quad \forall t \in\left[t_{k-1}, t_{k}\right), k \in N,
$$

for all $k \leq m$, where $k, m \in N$. We proceed to show that

$$
E V(x(t), t) \leq M_{m+1}\|\xi\|^{p} e^{-\lambda\left(t_{m+1}-t_{0}\right)}, \quad \forall t \in\left[t_{m}, t_{m+1}\right) .
$$


Suppose (3.41) is not true, set $\bar{t}=\inf \left\{t \in\left[t_{m}, t_{m+1}\right): E V(x(t), t)>M_{k}\|\xi\|^{p} e^{-\lambda\left(t_{m+1}-t_{0}\right)}\right\}$. By condition (ii),

$$
\begin{aligned}
E V\left(x\left(t_{m}\right), t_{m}\right) & \leq \rho d_{m} E V\left(x\left(t_{m}^{-}\right), t_{m}^{-}\right) \leq \rho M_{m+1}\|\xi\|^{p} e^{-\lambda\left(t_{m}-t_{0}\right)} \\
& <M_{m+1}\|\xi\|^{p} e^{-\lambda\left(t_{m+1}-t_{0}\right)} .
\end{aligned}
$$

From this, together with $E V(x(t), t)$ being continuous on $t \in\left[t_{m}, t_{m+1}\right)$, we know that $\bar{t} \in$ $\left(t_{m}, t_{m+1}\right)$ and

$$
E V(x(t), t)<E V(x(\bar{t}), \bar{t})=M_{m+1}\|\xi\|^{p} e^{-\lambda\left(t_{m+1}-t_{0}\right)}, \quad \forall t \in\left[t_{m}, \bar{t}\right) .
$$

Define $\underline{t}=\sup \left\{t \in\left[t_{0}, \bar{t}\right]: E V(x(t), t) \leq \rho M_{m+1}\|\xi\|^{p} e^{-\lambda\left(t_{m}-t_{0}\right)}\right\}$, then $\underline{t} \in\left[t_{m}, \bar{t}\right)$ and

$$
E V(x(t), t)>E V(x(\underline{t}), \underline{t})=\rho M_{m+1}\|\xi\|^{p} e^{-\lambda\left(t_{m}-t_{0}\right)}, \quad \forall t \in(\underline{t}, \bar{t}] .
$$

For $t \in[\underline{t}, \bar{t}]$ and $\theta \in[-\tau, 0]$, when $t+\theta \geq t_{m}$, then (3.44) implies that

$$
\begin{aligned}
E V(x(t+\theta), t+\theta) & \leq M_{m+1}\|\xi\|^{p} e^{-\lambda\left(t_{m+1}-t_{0}\right)} \\
& =\rho^{-1} e^{-\lambda\left(t_{m+1}-t_{m}\right)} E V(x(\underline{t}), \underline{t}) \\
& <q E V(x(\underline{t}), \underline{t}) .
\end{aligned}
$$

If $t+\theta<t_{m}$ for some $\theta \in[-\tau, 0)$, we assume that, without loss of generality, $t+\theta \in\left[t_{l}, t_{l+1}\right)$ for some $l \in N, l \leq m-1$, then from (3.41) and (3.44), we obtain

$$
\begin{aligned}
E V(x(t+\theta), t+\theta) & \leq M_{l+1}\|\xi\|^{p} e^{-\lambda\left(t_{l+1}-t_{0}\right)}<M_{l+1}\|\xi\|^{p} e^{-\lambda\left(t+\theta-t_{0}\right)} \\
& \leq M_{l+1} e^{\lambda \tau}\|\xi\|^{p} e^{-\lambda\left(t-t_{0}\right)} \leq \rho^{-1} e^{\lambda \tau} \frac{M_{l+1}}{M_{m+1}} E V(x(\underline{t}), \underline{t}) \\
& =\rho^{-1} e^{\lambda \tau} \hat{d}^{-1} E V(x(\underline{t}), \underline{t}) \leq q E V(x(\underline{t}), \underline{t}) .
\end{aligned}
$$

Therefore,

$$
E V(x(t+\theta), t+\theta)<q E V(x(t), t), \quad t \in[\underline{t}, \bar{t}], \theta \in[-\tau, 0] .
$$

The rest of the proof is similar to that of Theorem 3.2 and omitted here.

Remark 3.5. Let $\bar{u}$ and $\delta$ be positive constants. Assume that the conditions of Theorem 3.4 hold, function $u:\left[t_{0}, \infty\right) \rightarrow R_{+}$satisfies $\int_{t}^{t+\delta} u(s) d s \leq \bar{u} \delta$ and $\sup _{k \in \mathbb{N}}\left\{t_{k}-t_{k-1}\right\}=\delta<$ $-(\ln \rho /(\lambda+\bar{u}))$. Then Theorem 3.1 of [24] follows immediately.

Remark 3.6. It is not strictly required by condition (ii) of Theorem 3.4 that each impulse contributes to stabilize the system, as long as the overall contribution of the impulses are stabilizing. Without these $d_{k}$ (i.e., $d_{k} \equiv 1$ ), it is required that each impulse is a stabilizing factor $(\rho<1)$, which is more restrictive. 
Remark 3.7. It is clear that Theorems 3.2 and 3.4 allow the continuous dynamics of system (2.1) to be unstable, since the function $u(t)$, which characterizes the changing rate of $V(x(t), t)$ at $t$, is assumed to be nonnegative. Theorems 3.2 and 3.4 show that an unstable stochastic delay system can be successfully stabilized by impulses.

The following theorems show that the trivial solutions of system (2.1) are also almost surely exponentially stable, under some additional conditions.

Assumption 3.8. Suppose the impulsive instances $t_{k}$ satisfy

$$
\sup _{k \in N}\left\{t_{k}-t_{k-1}\right\}<\infty, \quad \inf _{k \in N}\left\{t_{k}-t_{k-1}\right\}>0 .
$$

Assumption 3.9. Assume that there is a constant $L>0$ such that, for all $(\phi, t) \in$ $P C_{q_{t}}^{p}\left([-\tau, 0] ; R^{n}\right) \times\left[t_{0}, \infty\right)$,

$$
E\left[|f(\phi, t)|^{p}+|g(\phi, t)|^{p}\right] \leq L \sup _{-\tau \leq \theta \leq 0} E|\phi(\theta)|
$$

Lemma 3.10 (see [23]). Let $p \geq 1$, and let Assumptions 3.8 and 3.9 hold. Then (3.31) implies that, for all $t \geq t_{0}$,

$$
\left|x\left(t ; \xi, t_{0}\right)\right| \leq C e^{-(\lambda / p)\left(t-t_{0}\right)}\|\xi\|^{p} \quad \text { a.s. }
$$

where $C$ is a positive constant. In other words, under Assumptions 3.8 and 3.9, the pth moment exponential stability implies the almost exponential stability for system (2.1).

By using Theorems 3.2 and 3.4 and Lemma 3.10, it is easy to show the following conclusions.

Theorem 3.11. Suppose that $p \geq 1$, Assumptions 3.8 and 3.9 and the same conditions as in Theorem 3.2 hold. Then the trivial solution of system (2.1) is also almost surely exponentially stable, with its Lyapunov exponent not greater than $-\lambda / p$.

Theorem 3.12. Suppose that $p \geq 1$, Assumptions 3.8 and 3.9 and the same conditions as in Theorem 3.4 hold. Then the trivial solution of system (2.1) is also almost surely exponentially stable, with its Lyapunov exponent not greater than $-\lambda / p$.

\section{An Example}

Example 4.1. Consider a scalar ISDDs of the form

$$
\begin{gathered}
d x(t)=x(t) d t+\frac{1}{4} \sqrt{x^{2}(t)+x^{2}(t-2)} d w(t), \quad t \neq t_{k}, t \geq t_{0} \\
\Delta x\left(t_{k}\right)=-0.4 x\left(t_{k}^{-}\right), \quad k \in N .
\end{gathered}
$$

It is easy to check that the corresponding system without impulses is not mean square exponentially stable. In fact, if $V(x, t)=x^{2}$, then it follows from the Ito formula that 


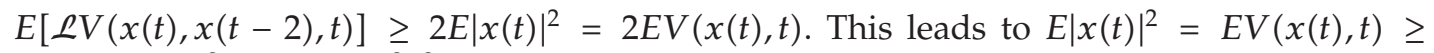
$E V(x(0), 0) e^{2 t}=E|x(0)|^{2} e^{2 t}$ for all $t \geq 0$. But, in the following, we will show that system (4.1) is mean square exponentially stable and almost exponentially stable.

If $V(x(t), t)=x^{2}$, then condition (i) of Theorem 3.2 holds with $c_{1}=c_{2}=1, p=2$, and condition (ii) holds with $d_{k}=0.36$. By calculating, we have $E[\mathcal{L V}(x(t), x(t-2), t)] \leq$ $(33 / 16) E V(x(t), t)+(1 / 16) E V(x(t-2), t)$. By taking $q=5, \lambda=0.5$, and $t_{k}-t_{k-1}=0.3$, it is easy to verify that condition (iii) of Theorem 3.2 is satisfied, which means system (4.1) is mean square exponentially stable. Applying Theorem 3.11, we can derive that system (4.1) is almost exponentially stable.

\section{Acknowledgments}

The authors are grateful to Editor Professor Josef Diblík and anonymous referees for their helpful comments and suggestions which have improved the quality of this paper. This work is supported by Natural Science Foundation of China (no. 10771001), Research Fund for Doctor Station of Ministry of Education of China (no. 20113401110001, no. 20103401120002), TIAN YUAN Series of Natural Science Foundation of China (no. 11126177), Key Natural Science Foundation (no. KJ2009A49), Talent Foundation (no. 05025104) of Anhui Province Education Department, 211 Project of Anhui University (no. KJJQ1101), Anhui Provincial Nature Science Foundation (no. 090416237, no. 1208085QA15), and Foundation for Young Talents in College of Anhui Province (no. 2012SQRL021).

\section{References}

[1] J. Baštinec, J. Diblík, D. Y. Khusainov, and A. Ryvolová, “Exponential stability and estimation of solutions of linear differential systems of neutral type with constant coefficients," Boundary Value Problems, vol. 2010, Article ID 956121, 20 pages, 2010.

[2] A.V. Shatyrko, D. Y. Khusainov, J. Diblík, J. Bastinec, and A. Ryvolova, "Estimates of perturbations of nonlinear indirect interval control system of neutral type," Journal of Automation and Information Sciences, vol. 43, no. 1, pp. 13-28, 2011.

[3] S. Peng and L. Yang, "Global exponential stability of impulsive functional differential equations via Razumikhin technique," Abstract and Applied Analysis, vol. 2010, Article ID 987372, 11 pages, 2010.

[4] J. Diblík and A. Zafer, "On stability of linear delay differential equations under Perron's condition," Abstract and Applied Analysis, vol. 2011, Article ID 134072, 9 pages, 2011.

[5] J. Diblík, D. Y. Khusainov, I. V. Grytsay, and Z. Šmarda, "Stability of nonlinear autonomous quadratic discrete systems in the critical case," Discrete Dynamics in Nature and Society, vol. 2010, Article ID 539087, 23 pages, 2010.

[6] J. Diblík, D. Ya. Khusainov, and I.V. Grytsay, "Stability investigation of nonlinear quadratic discrete dynamics systems in the critical case," Journal of Physics: Conference Series, vol. 96, no. 1, Article ID 012042, 2008.

[7] J. Luo, "Exponential stability for stochastic neutral partial functional differential equations," Journal of Mathematical Analysis and Applications, vol. 355, no. 1, pp. 414-425, 2009.

[8] I. A. Dzhalladova, J. Baštinec, J. Diblík, and D. Y. Khusainov, "Estimates of exponential stability for solutions of stochastic control systems with delay," Abstract and Applied Analysis, vol. 2011, Article ID 920412, 14 pages, 2011.

[9] S. Janković, J. Randjelović, and M. Jovanović, "Razumikhin-type exponential stability criteria of neutral stochastic functional differential equations," Journal of Mathematical Analysis and Applications, vol. 355, no. 2, pp. 811-820, 2009.

[10] Z. Yu, "Almost surely asymptotic stability of exact and numerical solutions for neutral stochastic pantograph equations," Abstract and Applied Analysis, vol. 2011, Article ID 143079, 14 pages, 2011.

[11] X. Mao, Stochastic Differential Equations and Applications, Horwood, Chichester, UK, 1997. 
[12] V. Lakshmikantham, D. D. Baĭnov, and P. S. Simeonov, Theory of Impulsive Differential Equations, vol. 6, World Scientific Publishing, Teaneck, NJ, USA, 1989.

[13] A. Lin and L. Hu, "Existence results for impulsive neutral stochastic functional integro-differential inclusions with nonlocal initial conditions," Computers $\mathcal{E}$ Mathematics with Applications, vol. 59, no. 1, pp. 64-73, 2010.

[14] B. Liu, "Stability of solutions for stochastic impulsive systems via comparison approach," IEEE Transactions on Automatic Control, vol. 53, no. 9, pp. 2128-2133, 2008.

[15] R. Sakthivel and J. Luo, "Asymptotic stability of nonlinear impulsive stochastic differential equations," Statistics E Probability Letters, vol. 79, no. 9, pp. 1219-1223, 2009.

[16] Q. Song and Z. Wang, "Stability analysis of impulsive stochastic Cohen-Grossberg neural networks with mixed time delays," Physica A, vol. 387, no. 13, pp. 3314-3326, 2008.

[17] X. Wang, Q. Guo, and D. Xu, "Exponential p-stability of impulsive stochastic Cohen-Grossberg neural networks with mixed delays," Mathematics and Computers in Simulation, vol. 79, no. 5, pp. 1698-1710, 2009.

[18] H. Wu and J. Sun, " $p$-moment stability of stochastic differential equations with impulsive jump and Markovian switching," Automatica, vol. 42, no. 10, pp. 1753-1759, 2006.

[19] L. Xu and D. Xu, "Mean square exponential stability of impulsive control stochastic system with time-varying delay," Physics Letters A, vol. 373, no. 3, pp. 328-333, 2009.

[20] L. Shen and J. Sun, " $p$-th moment exponential stability of stochastic differential equations with impulse effect," Science China Information Sciences, vol. 54, no. 8, pp. 1702-1711, 2011.

[21] P. Cheng, F. Deng, and X. Dai, "Razumikhin-type theorems for asymptotic stability of impulsive stochastic functional differential systems," Journal of Systems Science and Systems Engineering, vol. 19, no. 1, pp. 72-84, 2010.

[22] S. Peng and B. Jia, "Some criteria on $p$ th moment stability of impulsive stochastic functional differential equations," Statistics \& Probability Letters, vol. 80, no. 13-14, pp. 1085-1092, 2010.

[23] P. Cheng and F. Deng, "Global exponential stability of impulsive stochastic functional differential systems," Statistics E Probability Letters, vol. 80, no. 23-24, pp. 1854-1862, 2010.

[24] J. Liu, X. Liu, and W.-C. Xie, "Impulsive stabilization of stochastic functional differential equations," Applied Mathematics Letters, vol. 24, no. 3, pp. 264-269, 2011.

[25] J. Liu, X. Liu, and W.-C. Xie, "Existence and uniqueness results for impulsive hybrid stochastic delay systems," Communications on Applied Nonlinear Analysis, vol. 17, no. 3, pp. 37-53, 2010. 


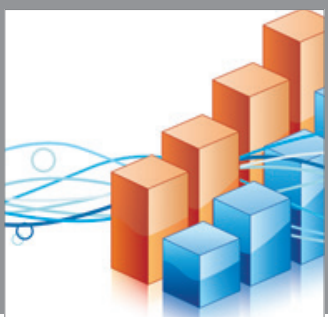

Advances in

Operations Research

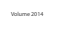

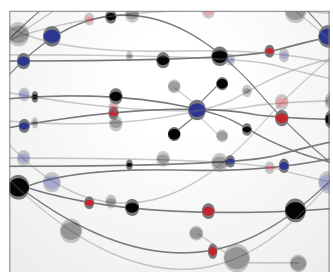

\section{The Scientific} World Journal
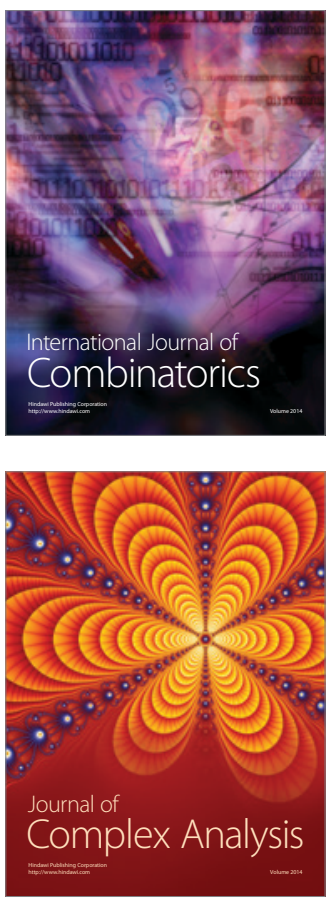

International Journal of

Mathematics and

Mathematical

Sciences
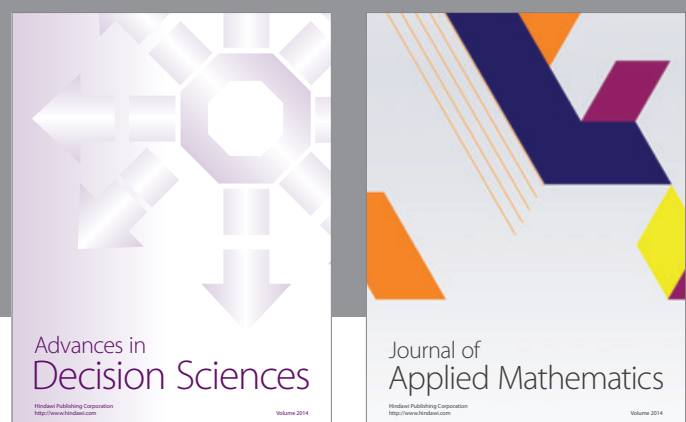

Journal of

Applied Mathematics
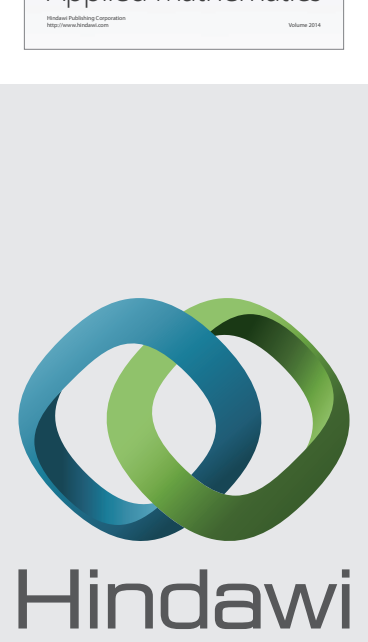

Submit your manuscripts at http://www.hindawi.com
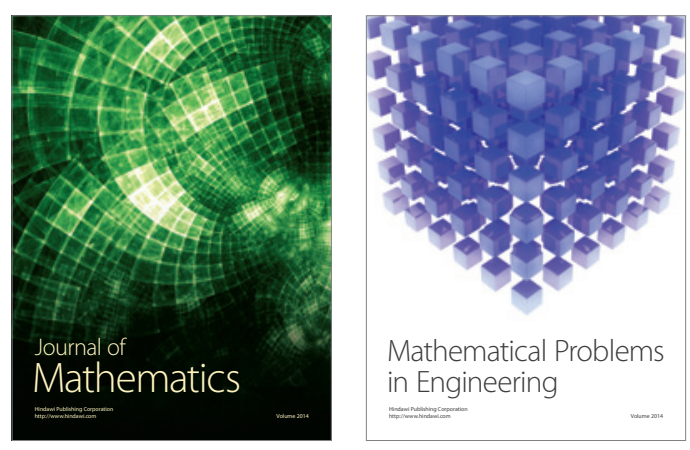

Mathematical Problems in Engineering


Journal of

Function Spaces




ournal of

Probability and Statistics

Promensencen
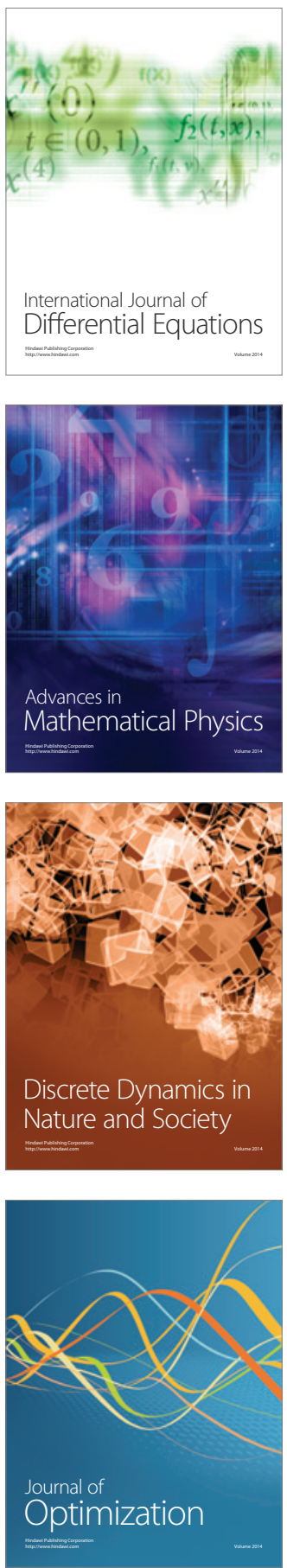